\title{
Replica Symmetry Breaking in Trajectories of a Driven Brownian Particle
}

\author{
$\operatorname{AUTHOR}(S)$ :
}

Ueda, Masahiko; Sasa, Shin-ichi

\section{CITATION:}

Ueda, Masahiko ... [et al]. Replica Symmetry Breaking in Trajectories of a Driven Brownian Particle. Physical review letters 2015, 115(8): 080605.

ISSUE DATE:

2015-08-21

URL:

http://hdl.handle.net/2433/201848

RIGHT:

C) 2015 American Physical Society. 


\title{
Replica Symmetry Breaking in Trajectories of a Driven Brownian Particle
}

\author{
Masahiko Ueda* and Shin-ichi Sasa ${ }^{\dagger}$ \\ Department of Physics, Kyoto University, Kyoto 606-8502, Japan
}

(Received 7 November 2014; published 21 August 2015)

\begin{abstract}
We study a Brownian particle passively driven by a field obeying the noisy Burgers' equation. We demonstrate that the system exhibits replica symmetry breaking in the path ensemble with the initial position of the particle being fixed. The key step of the proof is that the path ensemble with a modified boundary condition can be exactly mapped onto the canonical ensemble of directed polymers.
\end{abstract}

DOI: 10.1103/PhysRevLett.115.080605

PACS numbers: 05.40.-a, 05.70.Fh, 64.70.P-

Introduction.-Fluctuation and transportation in active environments have gradually attracted attention in recent studies of nonequilibrium physics and biophysics [1-8]. Among many problems, the prediction of the transportation properties of biological materials in living cells is a challenging problem in theoretical physics, and the possibility of such a prediction is a significant step in the control of cells [1-5]. In order to solve the problem, recalling the theory of Brownian motion in equilibrium environments [9], we seek for a universal principle for fluctuation and transportation in active environments by the analysis of trajectories of tracer particles. In particular, currently, it is significant to discover a key concept that is helpful in studying apparently complicated phenomena. We thus study the motion of a tracer particle in a simple system.

When we focus on the trajectories of a tracer particle, the most fundamental quantity is the displacement of the particle. For example, the mean-squared displacement as a function of time classifies diffusion behaviors such as superdiffusion [8,10,11] and subdiffusion [12,13]. More generally, the statistical properties of displacement during a finite time interval are characterized by the cumulant generating function of the displacement. This is often referred to as the dynamical free energy of displacement, which is associated with a statistical mechanical framework of trajectories $[14,15]$. Such a framework can be developed for other quantities such as activity [16-19] and Lyapunov exponent [20-22]. In this context, as a remarkable result, the study of the dynamical free energy of activity in glassy systems led to the discovery of the first-order transition, wherein dynamical heterogeneity is described as the coexistence of active and inactive phases in space-time at the first-order transition point. Since analysis of the dynamical free energy can reveal the singular nature of trajectories, it is useful to discover new phenomena associated with hidden singularities of the trajectories of a tracer particle in active environments.

In this Letter, we study the dynamical free energy of overlap. We here briefly review the concept of overlap in spin-glass theory [23]. Suppose that two independent and identical mean-field spin-glass systems (replicas) are prepared. Then, the distribution function of the overlap, which represents the similarity between the two spin configurations, takes a nontrivial form in the spin-glass phase, reflecting the existence of several stable configurations. This corresponds to replica symmetry breaking (RSB). The concept of RSB has so far been applied to information theory and computational complexity theory [24]. Here, we apply this method of RSB detection to the trajectories of two tracer particles [25]. That is, we prepare two independent particles and define the overlap as the similarity between the trajectories of both particles. We identify RSB by the existence of a nontrivial feature of the distribution function of the overlap. Therefore, the dynamical phase transition reported in this Letter is characterized by RSB in the path ensemble.

Concretely, we study an active environment obeying the noisy Burgers' equation [26]. The noisy Burgers' equation was introduced as a toy model of turbulence, and it is equivalent to the Kardar-Parisi-Zhang (KPZ) equation [27], which has been extensively studied [28-38]. In this study, we find that the overlap between the trajectories of two tracer particles obeys a nontrivial distribution, and we subsequently provide evidence to support the claim that this model exhibits RSB in the path ensemble.

Model.-Let $x(t)$ be the one-dimensional position of a Brownian particle at time $t \in[0, \tau]$. The particle is assumed to be passively driven by a velocity field $u(x, t)$. The motion of the particle is written as

$$
\dot{x}(t)=u(x(t), t)+\xi(t),
$$

where $\dot{x}(t) \equiv d x(t) / d t$ and $\xi(t)$ represents the thermal noise satisfying $\left\langle\xi(t) \xi\left(t^{\prime}\right)\right\rangle=2 D \delta\left(t-t^{\prime}\right)$ with the diffusion constant $D$ for the free particle. The velocity field is assumed to obey the noisy Burgers' equation. By setting $u=-\partial \phi / \partial x$, the equation for $\phi$ is expressed as the KPZ equation:

$$
\frac{\partial \phi}{\partial t}=\nu \frac{\partial^{2} \phi}{\partial x^{2}}+\frac{1}{2}\left(\frac{\partial \phi}{\partial x}\right)^{2}+v(x, t)
$$


where $v(x, t)$ represents zero-mean Gaussian white noise with variance $\left\langle v(x, t) v\left(x^{\prime}, t^{\prime}\right)\right\rangle=2 B \delta\left(x-x^{\prime}\right) \delta\left(t-t^{\prime}\right)$. More precisely, since the space-time Gaussian white noise is not properly defined, it is necessary to introduce a cutoff length $\Delta x$ in the space coordinate $[36,39]$. The derivatives in $x$ that appear in Eq. (2) are interpreted as simple differences. Accordingly, the field $u$ acting on the particle is evaluated by linear interpolation of $\phi$. Hereafter, we assume that all quantities are made dimensionless. We set $\Delta x=0.5$, and we find later that this choice of $\Delta x$ does not lead to any problems because all other length scales in this study are larger than $\Delta x$. For completeness, we also note the range of the particle position and the domain of the KPZ field. They are defined in a finite region with length $L$ under periodic boundary conditions, and $L$ is chosen to be sufficiently large such that the particle does not reach the boundaries within the observation time.

Before presenting our results, we briefly review previous studies involving this model. This model has been examined as an example of scalar turbulence [40], and it exhibits the remarkable phenomenon that noninteracting particles passively driven by the field cluster with time, wherein coalescence of valleys of the KPZ surface over time plays a key role [41]. Theoretically, renormalization group analysis has been performed for this model [42]. Furthermore, clusterization in the steady state has been detected by studying density-density correlation functions [43,44]. These previous studies indicate that particles in this model show a tendency towards localization. However, to the best of our knowledge, RSB in the path ensemble has never been reported.

Numerical result.-We first present the numerical simulation results of the model. In particular, we focus on the $D=\nu$ case, which corresponds to a fixed point of the renormalization group $[42,45]$. Time integration is performed by employing the simplest discretization method with $\Delta t=0.01$. We fix the initial position of the particle as $x(0)=L / 2$ with $L=10000$. The initial value of the field, $\phi(x, 0)$, is sampled from the stationary probability distribution of Eq. (2) [36]. Numerically, we obtain the distribution as the result of a sufficiently long time evolution of $\phi$. The parameter values are fixed as $D=\nu=1.0$ and $B=2.5$. Here, it should be noted that there are two sources of randomness, $\xi(t)$ and $v(x, t)$. In calculating an ensemble average of physical quantities, we first take the statistical average over $N_{1}$ histories of noise $\xi$ for one realization of $u(x, t)$, and we subsequently consider the configurational average over $N_{2}$ realizations of $v$. The convergence of quantities with respect to the choice of $\left(N_{1}, N_{2}\right)$ is carefully checked, and the numerical data presented below are obtained for $N_{1}=80000$ and $N_{2}=1000$.

We start with the observation of the trajectories for one realization of $u(x, t)$. As displayed in Fig. 1, each has the features of a typical trajectory of Brownian motion, while there exists a region in which several trajectories overlap.

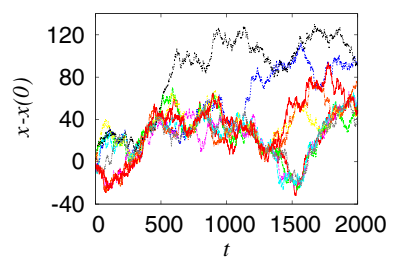

FIG. 1 (color online). Trajectories of a Brownian particle for one realization of $u(x, t)$. Ten samples are displayed.

We next proceed to quantify this observation. In the rest of this Letter, the ensemble average with respect to both $\xi(t)$ and $v(x, t)$ is taken for all physical quantities.

First, in the left panel of Fig. 2, the mean-squared displacement of $\sigma \equiv|x(t)-x(0)|$ as a function of $t$ is displayed. The particle behavior deviates from the normal diffusion type, and the anomalous diffusion $\left\langle\sigma^{2}\right\rangle \sim t^{4 / 3}$ is observed in the long time regime, as discussed in Refs. [41,42]. A distribution of $\sigma$ is also shown in the right panel of Fig. 2. Throughout this Letter, we use the same symbols (and colors) for such distribution functions with the same $t$. Since the width of $P(\sigma)$ increases with time $t$, localization phenomena are not detected by studying the one-particle behavior.

Next, the mean-squared value of the relative distance $d \equiv\left|x^{(1)}-x^{(2)}\right|$ for two trajectories $x^{(1)}$ and $x^{(2)}$ under the same $u(x, t)$ is investigated. The normal relative diffusion behavior $\left\langle d^{2}\right\rangle \sim t$ is clearly observed in the left panel of Fig. 3. On the other hand, as shown in the right panel of Fig. 3, the distribution of $d$ at time $t$, which is denoted by $P_{t}(d)$, is far from the Gaussian distribution that is obtained for free Brownian particles. As a characteristic feature of $P_{t}(d)$, it is observed that the value of $P_{t}(d)$ for $d<60$ does not change over time when $t>200$. The existence of such a time-independent behavior indicates that the interdistance of two particles is not significantly larger than a certain characteristic length. This indication provides one piece of quantitative evidence for a localization phenomenon. In our study, we estimated the characteristic length scale in this region as $d_{0}=4.2$ [46]. We remark here that such a
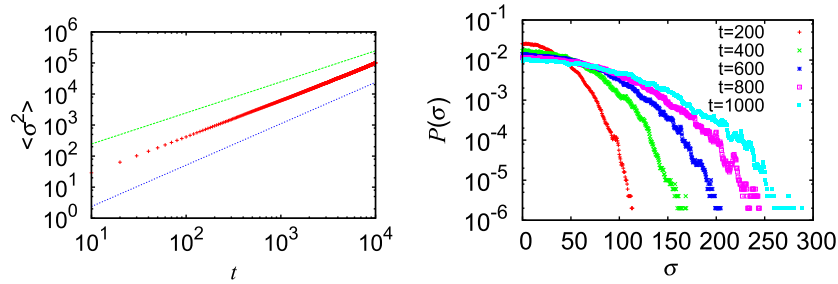

FIG. 2 (color online). The left panel shows the mean-squared displacement $\left\langle\sigma^{2}\right\rangle$ as a function of $t$ in a log-log plot. The straight lines represent the normal diffusion behavior $\left\langle\sigma^{2}\right\rangle \sim t$ and the anomalous diffusion behavior $\left\langle\sigma^{2}\right\rangle \sim t^{4 / 3}$, respectively. The right panel shows the distribution of $\sigma$ for various values of $t$. A logarithmic scale is used for the vertical axis. 

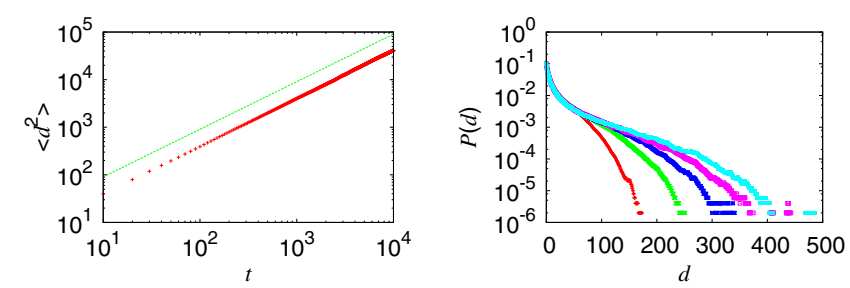

FIG. 3 (color online). The left panel shows the mean-squared relative distance $\left\langle d^{2}\right\rangle$ as a function of $t$ in a log-log plot. The straight line represents the normal diffusion behavior $\left\langle d^{2}\right\rangle \sim t$. The right panel shows the distribution of $d$ for various values of $t$. A logarithmic scale is used for the vertical axis.

diffusion scaling with a non-Gaussian distribution has attracted considerable research attention recently [4,52].

In order to characterize this phenomenon as a dynamical singularity in the path ensemble, we next use an analogy with the spin-glass theory. We consider the overlap defined by

$$
q \equiv \frac{1}{M} \sum_{j=1}^{M} \theta\left(\ell-\left|x^{(1)}(j \Delta t)-x^{(2)}(j \Delta t)\right|\right),
$$

for two trajectories $x^{(1)}$ and $x^{(2)}$ under the same $u(x, t)$. Here, $M \equiv t / \Delta t$ and we have introduced the length scale $\ell$ characterizing the localization. From the above discussion, $\ell$ should be close to $d_{0}$. For simplicity, we set $\ell=5$. In the left panel of Fig. 4, we show the distribution function of the overlap, $P(q)$, for different values of $t$. There are two peaks at $q=0$ and $q=q_{*}(t)$ for every $t$, which is a characteristic behavior observed in the one-step RSB (1RSB) [24]. The peak value at $q=0$ and the peak position $q_{*}(t)$ are shown in the right panels of Fig. 4. It appears that these approach finite values in the limit $t \rightarrow \infty$. In this work, we determine that RSB in the path ensemble occurs in the model under study.

The presence of two peaks in $P(q)$ indicates the coexistence of a high-overlap phase and a low-overlap phase in space-time. When we introduce the generating function of $q$ as $\psi(\epsilon) \equiv \lim _{\tau \rightarrow \infty} \log \left\langle e^{\tau \epsilon q}\right\rangle / \tau$, which is the dynamical free energy of overlap, the coexistence leads to a singularity in the dynamical free energy at $\epsilon=0$, which implies a dynamical phase transition. We note that such a dynamical phase transition can be described in terms of the path ensemble of two independent systems biased by
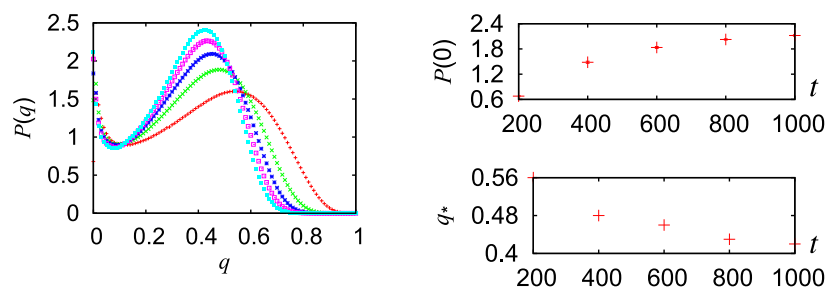

FIG. 4 (color online). Distribution of the overlap $q$ for various $t$ (left panel) and their peak values at $q=0$ and peak positions $q_{*}(t)$ (right panels). overlap. RSB in the trajectories indicates that the trajectories of two independent systems with a positive (zero) overlap are most weighted by an infinitely small positive (negative) bias $\epsilon$, which results in a singularity at $\epsilon=0$.

Analysis.-We present arguments to support the occurrence of RSB in the path ensemble. A key observation is that the ensemble of trajectories of the particle under a modified boundary condition is equivalent to the canonical ensemble of directed polymers with one end fixed that are subjected to a random potential. Explicitly, when we consider Eqs. (1) and (2) with boundary conditions $x(\tau)=$ $x_{0}=L / 2$ and $\phi(x, 0)=0$, the path probability density of the particle is written as

$$
\mathcal{P}\left[x \mid x(\tau)=x_{0}\right]=\frac{1}{Z_{\mathrm{DP}}} e^{-(1 / 4 D) \int_{0}^{\tau} d t\left[\dot{x}(t)^{2}-2 v(x(t), t)\right]},
$$

where $Z_{\mathrm{DP}}$ represents the normalization constant. The proof is as follows. We first write the Onsager-Machlup expression of the path probability density as

$$
\begin{aligned}
\mathcal{P}\left[x \mid x(\tau)=x_{0}\right]= & \frac{1}{Z_{0}} e^{-(1 / 4 D) \int_{0}^{\tau} d t[\dot{x}(t)+(\partial \phi / \partial x)(x(t), t)]^{2}} \\
& \times e^{-(1 / 2) \int_{0}^{\tau} d t\left(\partial^{2} \phi / \partial x^{2}\right)(x(t), t)}
\end{aligned}
$$

where $Z_{0}$ denotes the normalization constant, and the second term in the exponential arises from the transformation from $[\xi(t)]_{t=0}^{\tau}$ to $[x(t)]_{t=0}^{\tau}$, with $x(\tau)$ fixed [53]. We subsequently rewrite Eq. (5) using Eq. (2) as

$$
\begin{aligned}
\mathcal{P}[x \mid & \left.x(\tau)=x_{0}\right] \\
= & \frac{1}{Z_{0}} e^{-(1 / 4 D) \int_{0}^{\tau} d t\left[\dot{x}(t)^{2}+2(d / d t) \phi(x(t), t)-2(\partial \phi / \partial t)(x(t), t)\right]} \\
& \times e^{-(1 / 4 D) \int_{0}^{\tau} d t\left\{[(\partial \phi / \partial x)(x(t), t)]^{2}+2 D\left(\partial^{2} \phi / \partial x^{2}\right)(x(t), t)\right\}} \\
= & \frac{1}{Z_{0}} e^{-(1 / 2 D)[\phi(x(\tau), \tau)-\phi(x(0), 0)]-(1 / 4 D) \int_{0}^{\tau} d t\left[\dot{x}(t)^{2}-2 v(x(t), t)\right]} \\
= & \frac{1}{Z_{0}} e^{-(1 / 2 D) \phi\left(x_{0}, \tau\right)-(1 / 4 D) \int_{0}^{\tau} d t\left[\dot{x}(t)^{2}-2 v(x(t), t)\right]}
\end{aligned}
$$

Since the first term of the exponential can be absorbed into the normalization constant, we obtain Eq. (4).

The statistical model (4) has been extensively studied [36,54-62]. It has been shown that the system is in the frozen phase for $B>0$ and $D<\infty$. The most important result here is that the replica symmetry of the system is broken [54,55], which is obtained by the replica Bethe ansatz calculation and the numerical calculation of the transfer matrix for a discretized model. Thus, the equivalence demonstrated above implies that the Langevin model with $x(\tau)$ fixed also exhibits RSB.

Therefore, if the particle behavior in the time region $1 \ll$ $t \ll \tau$ is independent of $x(0)$ and $x(\tau)$, the original model with $x(0)$ fixed (which is easily prepared in experiments) 
can be shown to exhibit RSB. Although the validity of the independence of $x(0)$ and $x(\tau)$ is not assured, we can verify it in numerical simulations. That is, we numerically investigate the modified system with $x(\tau)$ fixed and compare the results with those obtained for the original model [63]. In the left panel of Fig. 5, we show $P(q)$ for the modified system. This result is nearly identical to that of the original case. The peak value and the nontrivial peak position also behave in the same way as in the original model, as seen in the right panels of Fig. 5. Thus, we can confidently conclude that $P(q)$ is independent of $x(0)$ and $x(\tau)$, and this implies that the original model definitely exhibits RSB. See Ref. [64] for discussions on the type of RSB.

At the end of our analysis, we attempt to provide a physical picture of RSB in the path ensemble. In Ref. [41], it was found for the $D=0$ case that valleys (local minima) of the velocity potential $\phi$ move continuously and eventually coalesce. Therefore, when $D=0$, independent particles with slightly different initial conditions cluster in a specific valley, which leads to $P(q)$ concentrated on $q=1$. However, in our model with $D>0$, a particle can escape from one valley of $\phi$ to another valley due to thermal noise $\xi$. We subsequently explain that this activation effect is too weak to remove the concentration of $P(q)$ on a finite overlap. First, it has been known that the steady-state distribution of the KPZ equation is equivalent to the probability distribution of a random potential field in the continuous version of the Sinai model [65], where ultraslow diffusion $\left\langle x(t)^{2}\right\rangle \sim(\log t)^{4}$ is observed. Now, let us suppose that we observe the distance of two independent particles with different thermal noises during a time interval $t$. The distance would be $\mathcal{O}\left((\log t)^{2}\right)$ for large $t$ if the potential were frozen. On the other hand, particles whose distance from each other is within a length scale $l_{\text {co }}$ cluster in one valley by coalescences of valleys, where $l_{\text {co }}$ is expected to be $\mathcal{O}\left(t^{\alpha}\right)$ with some $\alpha>0$ for large $t$ because it is determined by the diffusive nature of motion of the valleys. Therefore, from $t^{\alpha} \gg(\log t)^{2}$ for large $t$, we conclude that the phenomenon observed in the system with $D=0$ is robust to thermal noise. The importance of
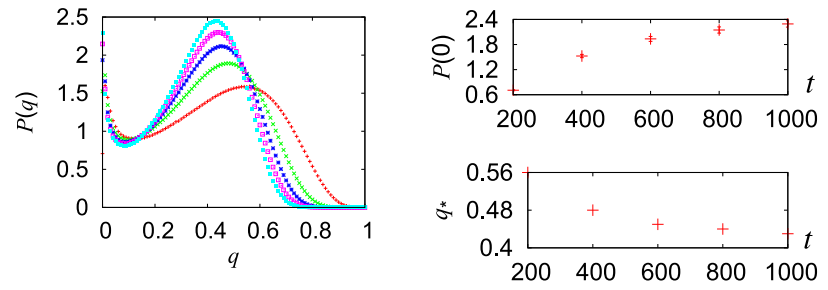

FIG. 5 (color online). Statistical properties of the ensemble (5). The distribution of the overlap, $P(q)$, for various $t^{\prime} \equiv \tau-t$ are plotted in the left panel, and their peak values at $q=0$ and peak positions $q_{*}\left(t^{\prime}\right)$ are displayed in the right panels. The symbols and color labels in the graph with $t^{\prime}$ are the same as those in the graph with $t$ in Fig. 4. the coalescence of valleys can be understood from the absence of RSB for the case where particles are driven by a field obeying the Edwards-Wilkinson (EW) equation [66], because the steady-state probability distribution of the potential field for the EW equation is the same as that for the KPZ equation, while the valleys of the potential do not coalesce.

Concluding remarks.-We discuss previous studies related to our results. It has been known that two independent and identical dynamical systems exhibit mutual attraction of trajectories with slightly different initial conditions when they are subject to a common noise. Examples include synchronization of two independent oscillators driven by a common noise $[67,68]$, and aggregation of independent particles driven by a common Gaussian random velocity field $[69,70]$ or a common spatiotemporal chaotic field [71]. A more recent work on diffusive fluctuating hydrodynamics reports a similar phenomenon through the analysis of the dynamical free energy of the Lyapunov exponent [22]. These phenomena are similar to that studied in our Letter in that attraction of trajectories in two independent and identical systems is concerned. One can interpret that a common disordered velocity field in our study corresponds to a common noise in previous studies. Although this interpretation is reasonable, the two systems in our study are subject to independent thermal noises in addition to a common velocity field, which makes a contrast to previous studies. We also remark that mutual attraction of trajectories in two independent systems was observed in our system without thermal noise [41]. We emphasize that to find the common-noise-induced attraction robust against independent noises is a highly nontrivial problem, which corresponds to the discovery of a phase transition at finite temperature for a symmetry breaking at $T=0$ in equilibrium statistical mechanics. The main achievement of our study is that we solve this problem by using the concept of RSB.

In sum, we have demonstrated RSB in trajectories for a tracer particle passively driven by a field obeying the noisy Burgers' equation. In this model, two tracer particles driven by a common velocity field are always close to each other when they freeze to the same trajectory, while they become separated from each other with time when each particle freezes to a different trajectory. These two cases correspond to two peaks in $P(q)$ at $q=q_{*}$ and $q=0$, respectively. Such a pathological transportation property is detected only by the observation of $P(q)$. We believe that the dynamical free energy of overlap will be a useful tool to characterize a singularity of trajectories for a wide class of phenomena including chemical networks [72] and cell differentiation [73,74].

The authors thank T. Nemoto for the useful discussions, particularly regarding the derivation of Eq. (4). They also thank F. van Wijland and É. Fodor for their valuable comments. The present study was supported by KAKENHI 
(Grants No. 25103002 and No. 26610115), a Grant-in-Aid for JSPS Fellows (Grant No. 2681), and by the JSPS Coreto-Core program "Non-equilibrium dynamics of soft matter and information."

*ueda@ton.scphys.kyoto-u.ac.jp

†sasa@scphys.kyoto-u.ac.jp

[1] M. J. Saxton and K. Jacobson, Annu. Rev. Biophys. Biomol. Struct. 26, 373 (1997).

[2] H. Ewers, A. E. Smith, I. F. Sbalzarini, H. Lilie, P. Koumoutsakos, and A. Helenius, Proc. Natl. Acad. Sci. U.S.A. 102, 15110 (2005).

[3] P. Bursac, G. Lenormand, B. Fabry, M. Oliver, D. A. Weitz, V. Viasnoff, J. P. Butler, and J. J. Fredberg, Nat. Mater. 4, 557 (2005).

[4] B. Wang, S. M. Anthony, S. C. Bae, and S. Granick, Proc. Natl. Acad. Sci. U.S.A. 106, 15160 (2009).

[5] M. Guo, A. J. Ehrlicher, M. H. Jensen, M. Renz, J. R. Moore, R. D. Goldman, J. Lippincott-Schwartz, F. C. Mackintosh, and D. A. Weitz, Cell 158, 822 (2014).

[6] X.-L. Wu and A. Libchaber, Phys. Rev. Lett. 84, 3017 (2000).

[7] D. Mizuno, C. Tardin, C. F. Schmidt, and F. C. MacKintosh, Science 315, 370 (2007).

[8] B. Liu and J. Goree, Phys. Rev. Lett. 100, 055003 (2008).

[9] A. Einstein, Investigations on the Theory of the Brownian Movement (Dover Publications, New York, 1956).

[10] J.-P. Bouchaud, A. Georges, J. Koplik, A. Provata, and S. Redner, Phys. Rev. Lett. 64, 2503 (1990).

[11] E. Baskin and A. Iomin, Phys. Rev. Lett. 93, 120603 (2004).

[12] M. Weiss, M. Elsner, F. Kartberg, and T. Nilsson, Biophys. J. 87, 3518 (2004).

[13] I. Y. Wong, M. L. Gardel, D. R. Reichman, E. R. Weeks, M. T. Valentine, A. R. Bausch, and D. A. Weitz, Phys. Rev. Lett. 92, 178101 (2004).

[14] C. Beck and F. Schögl, Thermodynamics of Chaotic Systems: An Introduction (Cambridge University Press, Cambridge, England, 1993).

[15] M. Merolle, J. P. Garrahan, and D. Chandler, Proc. Natl. Acad. Sci. U.S.A. 102, 10837 (2005).

[16] J. P. Garrahan, R. L. Jack, V. Lecomte, E. Pitard, K. van Duijvendijk, and F. van Wijland, Phys. Rev. Lett. 98, 195702 (2007).

[17] J. P. Garrahan, R. L. Jack, V. Lecomte, E. Pitard, K. van Duijvendijk, and F. van Wijland, J. Phys. A 42, 075007 (2009).

[18] L. O. Hedges, R. L. Jack, J. P. Garrahan, and D. Chandler, Science 323, 1309 (2009).

[19] R. L. Jack and J. P. Garrahan, Phys. Rev. E 81, 01111 (2010).

[20] C. Giardina, J. Kurchan, V. Lecomte, and J. Tailleur, J. Stat. Phys. 145, 787 (2011).

[21] T. Laffargue, K. D. N. T. Lam, J. Kurchan, and J. Tailleur, J. Phys. A 46, 254002 (2013).

[22] T. Laffargue, P. Sollich, J. Tailleur, and F. van Wijland, Europhys. Lett. 110, 10006 (2015).

[23] M. Mézard, G. Parisi, and M. A. Virasoro, Spin Glass Theory and Beyond (World Scientific, Singapore, 1987).
[24] M. Mézard and A. Montanari, Information, Physics and Computation (Oxford University Press, Oxford, 2009).

[25] It should be noted that a similar attempt was made for globally coupled logistic map models in order to detect the existence of many attractors in S. C. Manrubia and A. S. Mikhailov, Europhys. Lett. 53, 451 (2001) and S.C. Manrubia, U. Bastolla, and A. S. Mikhailov, Eur. Phys. J. B 23, 497 (2001).

[26] J. Bec and K. Khanin, Phys. Rep. 447, 1 (2007).

[27] M. Kardar, G. Parisi, and Y.-C. Zhang, Phys. Rev. Lett. 56, 889 (1986).

[28] D. Forster, D. R. Nelson, and M. J. Stephen, Phys. Rev. A 16, 732 (1977).

[29] E. Medina, T. Hwa, M. Kardar, and Y.-C. Zhang, Phys. Rev. A 39, 3053 (1989).

[30] J. Krug, P. Meakin, and T. Halpin-Healy, Phys. Rev. A 45, 638 (1992).

[31] J. P. Bouchaud, M. Mézard, and G. Parisi, Phys. Rev. E 52, 3656 (1995).

[32] L. Bertini and G. Giacomin, Commun. Math. Phys. 183, 571 (1997).

[33] L. Canet, H. Chaté, B. Delamotte, and N. Wschebor, Phys. Rev. Lett. 104, 150601 (2010).

[34] T. Sasamoto and H. Spohn, Phys. Rev. Lett. 104, 230602 (2010).

[35] P. Calabrese and P. Le Doussal, Phys. Rev. Lett. 106, 250603 (2011).

[36] T. Halpin-Healy and Y.-C. Zhang, Phys. Rep. 254, 215 (1995).

[37] J. Maunuksela, M. Myllys, O.-P. Kahkonen, J. Timonen, N. Provatas, M. J. Alava, and T. Ala-Nissila, Phys. Rev. Lett. 79, 1515 (1997).

[38] K. A. Takeuchi, M. Sano, T. Sasamoto, and H. Spohn, Sci. Rep. 1, 34 (2011).

[39] Y.-C. Zhang, Physica (Amsterdam) 170A, 1 (1990).

[40] B. I. Shraiman and E. D. Siggia, Nature (London) 405, 639 (2000).

[41] C.-S. Chin, Phys. Rev. E 66, 021104 (2002).

[42] B. Drossel and M. Kardar, Phys. Rev. B 66, 195414 (2002).

[43] A. Nagar, M. Barma, and S. N. Majumdar, Phys. Rev. Lett. 94, 240601 (2005).

[44] A. Nagar, S. N. Majumdar, and M. Barma, Phys. Rev. E 74, 021124 (2006).

[45] D. Ertaş and M. Kardar, Phys. Rev. E 48, 1228 (1993).

[46] See Supplemental Material at http://link.aps.org/ supplemental/10.1103/PhysRevLett.115.080605, which includes Refs. [46-50], for the estimation of the length scale.

[47] D. A. Huse and D. S. Fisher, J. Phys. A 20, L997 (1987).

[48] G. Parisi and M. A. Virasoro, J. Phys. (Paris) 50, 3317 (1989).

[49] R. L. Jack and P. Sollich, Prog. Theor. Phys. Suppl. 184, 304 (2010).

[50] T. Nemoto and S.-i. Sasa, Phys. Rev. E 84, 061113 (2011).

[51] S. F. Edwards and D. R. Wilkinson, Proc. R. Soc. A 381, 17 (1982).

[52] B. Wang, J. Kuo, S. C. Bae, and S. Granick, Nat. Mater. 11, 481 (2012).

[53] See Supplemental Material at http://link.aps.org/ supplemental/10.1103/PhysRevLett.115.080605, which includes Refs. [46-50], for the derivation of Eq. (5). 
[54] G. Parisi, J. Phys. (Paris) 51, 1595 (1990).

[55] M. Mézard, J. Phys. (Paris) 51, 1831 (1990).

[56] M. Kardar and Y.-C. Zhang, Phys. Rev. Lett. 58, 2087 (1987).

[57] M. Kardar, Nucl. Phys. B290, 582 (1987).

[58] J. P. Bouchaud and H. Orland, J. Stat. Phys. 61, 877 (1990).

[59] T. Hwa and D. S. Fisher, Phys. Rev. B 49, 3136 (1994).

[60] H. Yoshino, J. Phys. A 29, 1421 (1996).

[61] É. Brunet and B. Derrida, Phys. Rev. E 61, 6789 (2000).

[62] P. Calabrese, P. Le Doussal, and A. Rosso, Europhys. Lett. 90, 20002 (2010).

[63] See Supplemental Material at http://link.aps.org/ supplemental/10.1103/PhysRevLett.115.080605, which includes Refs. [46-50], for a numerical method for preparing the path ensemble (5).

[64] See Supplemental Material at http://link.aps.org/ supplemental/10.1103/PhysRevLett.115.080605, which includes Refs. [46-50], for the type of RSB of our model.

[65] J. P. Bouchaud and A. Georges, Phys. Rep. 195, 127 (1990).
[66] See Supplemental Material at http://link.aps.org/ supplemental/10.1103/PhysRevLett.115.080605, which includes Refs. [46-50], for results for the EdwardsWilkinson equation.

[67] S. Boccaletti, J. Kurths, G. Osipov, D. L. Valladares, and C. S. Zhou, Phys. Rep. 366, 1 (2002).

[68] J. N. Teramae and D. Tanaka, Phys. Rev. Lett. 93, 204103 (2004).

[69] J. M. Deutsch, J. Phys. A 18, 1449 (1985).

[70] M. Wilkinson and B. Mehlig, Phys. Rev. E 68, 040101(R) (2003).

[71] T. Bohr and A. Pikovsky, Phys. Rev. Lett. 70, 2892 (1993).

[72] S. A. Kauffman, J. Theor. Biol. 22, 437 (1969).

[73] Z. S. Singer, J. Yong, J. Tischler, J. A. Hackett, A. Altinok, M. A. Surani, L. Cai, and M. B. Elowitz, Mol. Cell 55, 319 (2014).

[74] K. Kaneko, Life: An Introduction to Complex Systems Biology (Springer, New York, 2006). 